\title{
Synchrotron emission in molecular cloud cores: the SKA view (Corrigendum)
}

\author{
Marco Padovani and Daniele Galli
}

\begin{abstract}
INAF-Osservatorio Astrofisico di Arcetri, Largo E. Fermi 5, 50125 Firenze, Italy
\end{abstract} e-mail: padovani@arcetri.astro.it, galli@arcetri.astro.it

A\&A 620, L4 (2018), https: //doi .org/10 . 1051/0004-6361/201834222

Key words. ISM: clouds - ISM: magnetic fields - cosmic rays - errata, addenda

The specific intensity (Eq. (5)) has not been correctly evaluated $^{1}$. This affects the computation of the flux densities, whose correct trends are shown in the new versions of Figs. 3 and 4 . We recovered the signal-to-noise ratios $(\mathrm{S} / \mathrm{Ns})$ quoted in the paper by assuming a magnetic field strength $B_{0}$ larger by a factor of $2.74-4.20,2.02-2.72$, and 1.55-1.80 than originally assumed for models A, B, and C, respectively (the lower and upper values corresponding to $\kappa=0.5$ and $\kappa=0.7$, respectively). The conclusions of the paper are unaffected.

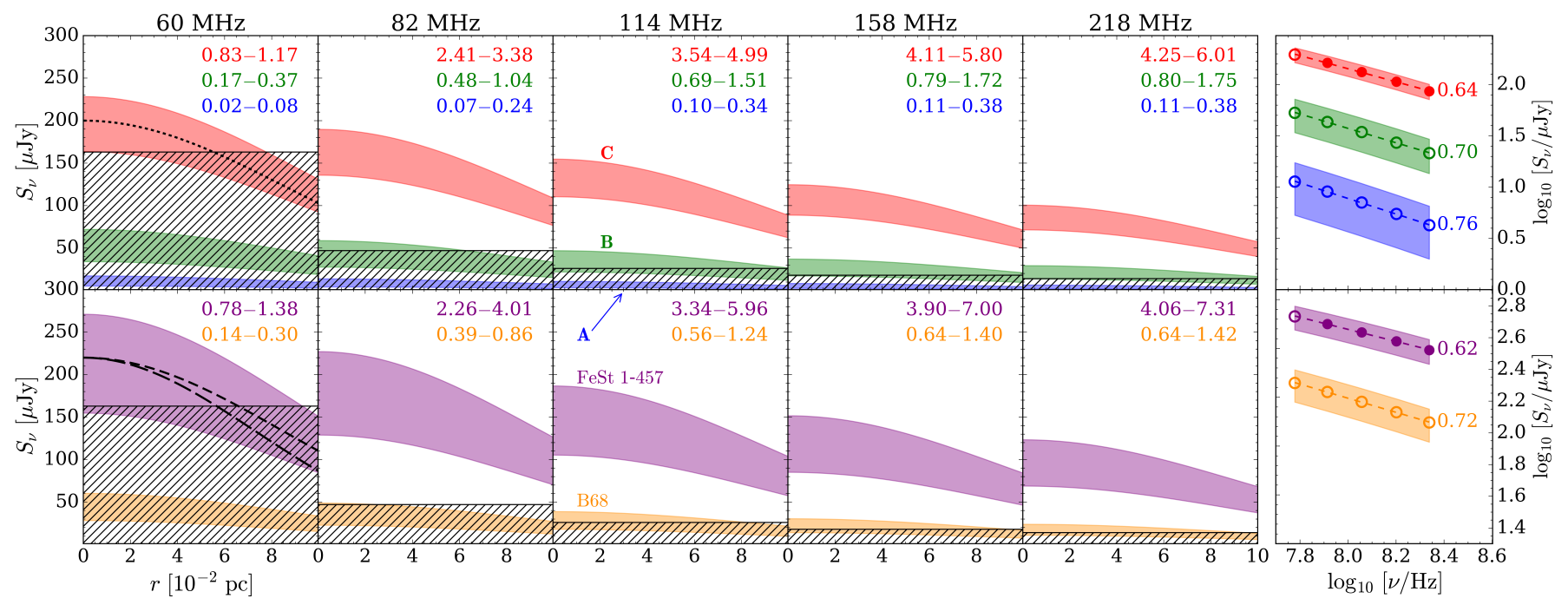

Fig. 3. Radial flux density profiles for the starless core models described in Sect. 5.1 (upper row) and for B68 and FeSt 1-457 (lower row, see Sect. 5.2). The observing frequency is shown in black at the top of each column, while numbers in the upper-right corner of each subplot represent the radius-averaged $\mathrm{S} / \mathrm{N}$ for the two values of $\kappa(0.5$ and 0.7 for models $\mathrm{A}, \mathrm{B}, \mathrm{C}$, and B68, and 0.68 and 0.88 for FeSt 1-457, see Eq. (9)). Shaded areas encompass the curves obtained with Eq. (9) by using the two values of $\kappa$ (see Fig. 2 for colour-coding). The telescope beam is shown in the leftmost column for models A, B, and C (dotted black line, 300"), B68 (short-dashed black line, 330"), and FeSt 1-457 (long-dashed black line, $\left.284^{\prime \prime}\right)$. Hatched areas display SKA sensitivities for one hour of integration at different frequencies. The two panels on the right side show the flux density as a function of frequency. Empty (solid) circles refer to an $\mathrm{S} / \mathrm{N}$ smaller (larger) than 3, respectively. The spectral index $\alpha$ is shown on the right of each curve.

\footnotetext{
1 We thank Alexei Ivlev for pointing out this mistake.
} 
A\&A 643, C1 (2020)

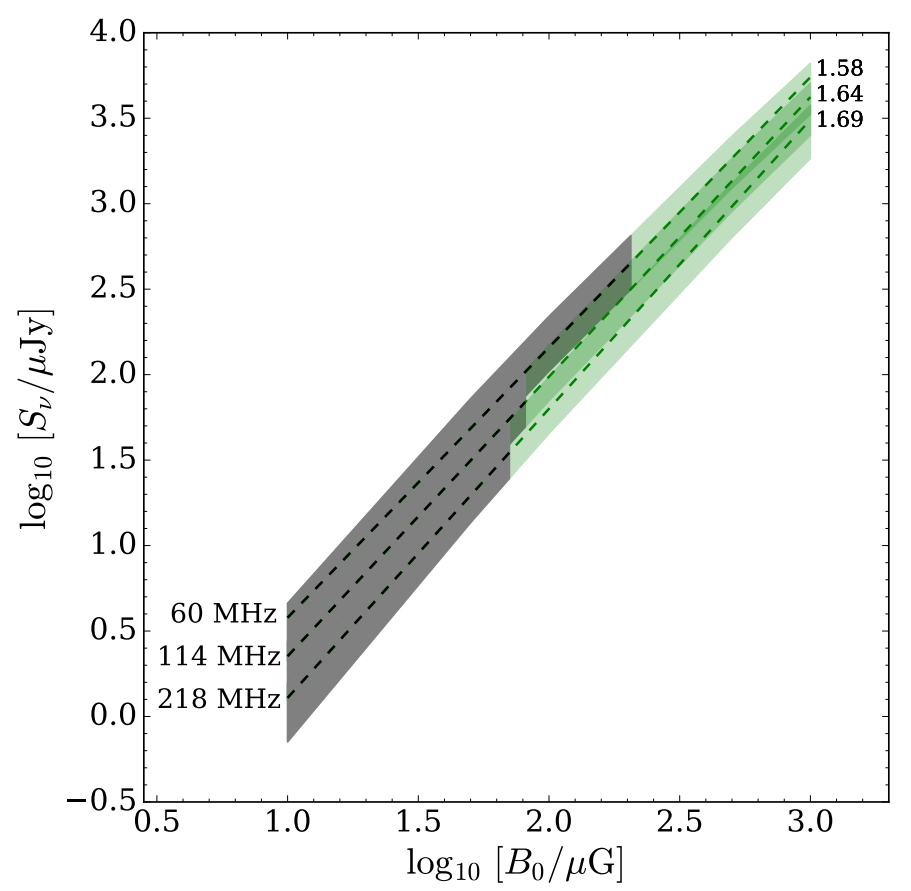

Fig. 4. Flux density at 60,114, and $218 \mathrm{MHz}$ (lower-left labels) as a function of magnetic field strength $B_{0}$ for model B (see Sect. 5.1). Green shaded areas encompass the curves obtained with Eq. (9) by using $\kappa=$ 0.5 and 0.7. Grey areas correspond to an $S / N<3$ and dashed lines are power-law fits of $S_{v} \propto B_{0}^{\delta}$ with $\delta$ listed in the upper-right corner. 